vol. 29 - $n^{\circ} 1 \mid 2013$

Mémoires et migrations en Afrique de l'Ouest et en

France

\title{
Histoire orale à Matonge (Bruxelles) : un miroir postcolonial
}

Oral History in Matonge (Brussels): A Post-colonial Mirror

Historia oral en Matonge (Bruselas): un espejo postcolonial

\section{Sarah Demart}

\section{OpenEdition}

Journals

\section{Édition électronique}

URL : https://journals.openedition.org/remi/6323

DOI : $10.4000 /$ remi.6323

ISSN : $1777-5418$

Éditeur

Université de Poitiers

\section{Édition imprimée}

Date de publication : 1 mars 2013

Pagination : 133-155

ISBN : 979-10-90426-07-8

ISSN : 0765-0752

\section{Référence électronique}

Sarah Demart, « Histoire orale à Matonge (Bruxelles) : un miroir postcolonial », Revue européenne des migrations internationales [En ligne], vol. 29 - $n^{\circ} 1$ | 2013, mis en ligne le 01 mars 2016, consulté le 14 avril 2022. URL : http://journals.openedition.org/remi/6323 ; DOI : https://doi.org/10.4000/remi.6323 


\section{Histoire orale à Matonge (Bruxelles) : un miroir postcolonial}

\section{Sarah Demart ${ }^{1}$}

\section{Introduction : un lieu dans la ville}

Le 30 mars 2010, le "Carrefour " ferme. Qu'on se le dise! Le Vieux Henri et Ma Hono remettent leur commerce. "Mère " est fatiguée. II y a cet escalier, entre la cuisine au sous-sol et la salle, que ses genoux ne supportent plus. Et puis c'est la crise, les clients ne consomment plus comme avant. Quelques habitués sont venus pour ce dernier soir, pour assister à la transaction, pour dire au revoir. II n'y a pas grand monde, c'est la semaine de Pâques, certaines clientes ou devraiton dire, habituées, amies, sœurs ont des activités à l'Église ou font carême. Et puis, c'est durant toute la semaine que les clients se sont succédé... Depuis des semaines, on se demande quel sera désormais notre lieu $d^{\prime}$ " atterrissage " à Matonge.

Matonge, du nom d'un quartier de Kinshasa ${ }^{2}$, est constitué de quelques rues autour de la chaussée de Wavre et des galeries d'Ixelles, à Bruxelles (commune d'lxelles), métro Porte de Namur, non loin de l'ambassade de la République démocratique du Congo (RDC), des institutions européennes et du très cossu quartier Louise. Aux côtés des ngandas ${ }^{3}$ et restaurants, on y trouve des magasins de pagnes, de chaussures, de sacs, de perruques ou de tissages. Les salons de coiffure pour hommes, femmes ou enfants, sont pléthores, de même que les call shops, les cybercafés et les bijouteries. Jusqu'il y a peu, un centre culturel organisait aussi régulièrement des rencontres littéraires, des conférences et des expositions.

Si la " griffe " du quartier est congolaise à l'instar de l'arrêt de bus (chaussée d'Ixelles, à la sortie du métro porte de Namur) mentionnant « Matongé-Porte de Namur " et de la gigantesque toile du peintre congolais Cheri Samba (Jewsiewicki, 2003) représentant, au sommet d'un bâtiment, la vie de Matonge

\footnotetext{
1 Chargée de recherches F.R.S.-FNRS, Centre d'Études de I'Ethnicité et des Migrations (CEDEM), ISHS - Bât. 31 - 7, bd du Rectorat, Université de Liège, 4000 Liège, Belgique ; sarah.demart@ulg.ac.be

2 Le quartier Matonge est un quartier très animé de Kinshasa où I'on va notamment chercher les dernières nouveautés musicales et vestimentaires, rencontrer ceux qui viennent d'Europe, les "Parisiens ", les "Belgicains ", les "Mikilistes " ou encore les célébrités locales (musicales ou sportives).

3 Lieux de vie, café, bar avec petite restauration.
} 
et ses personnalités ${ }^{4}$, le quartier n'est pas pour autant une enclave ethnique. En témoignent les plus de quarante nationalités coexistant dans cet espace (Corijn, 2004) ainsi que le nombre considérable d'établissements non " africains ". Outre les commerces tenus par des commerçants d'origine pakistanaise et libanaise, on trouve un Brico, un fast-food de la chaîne Hector, des agences de voyage et d'envoi de colis, une agence western-union, des restaurants asiatiques, des boutiques de vin, d'objets d'arts africains, etc. Un cinéma alternatif et un centre culturel, programmant des artistes africains, participent également de la vie de ce quartier, bien que la clientèle soit majoritairement blanche ${ }^{5}$. Selon la police, le quartier se singularise aussi par les nombreuses sociétés, toutes origines confondues $^{6}$, qui y ont leur siège social.

Mais pour les Congolais, Matonge n'est pas, et n'a jamais été, un lieu de résidence ${ }^{7}$. Espace de commerce, de rencontre et de festivité, Matonge est avant tout un lieu de passages. En ce mois de mars 2010, les " mamans " d'un certain âge ayant connu l'Europe dans les années 1960 et 1970 reprochent à la patronne du "Carrefour " de les " abandonner ": "Qu'est-ce qu'on va devenir nous maintenant? ". Elles ne sont pas les seules à se sentir " perdues " malgré l'offre foisonnante de ngandas à Matonge. Quelques jours après la remise de l'établissement, c'est ce groupe d'hommes habitués à se retrouver chez le vieux Henri qui erre. L'un est biologiste, l'autre juriste, celui-là économiste, celui-ci vétérinaire. Tshisekedistes ${ }^{8}$, ils aimaient bien se rendre au "Carrefour " dont le patron, malgré son nationalisme lumumbiste ${ }^{9}$ patenté, est resté un "vrai Muluba ${ }^{10}$ ". De façon paradoxale, il n'hésitait pas à se fâcher, voire à chasser ses propres clients lorsqu'il désapprouvait leurs opinions politiques, en particulier leur tribalisme, jusqu'à chasser son propre oncle : "Imaginer, chasser son oncle maternel, dans nos traditions africaines... ". C'est vrai, cela aussi a réduit la clientèle, même si le Vieux reste un monument.

Associé à la constitution de ce quartier, il est l'une de ses mémoires les plus vivantes. Pendant dix-sept ans, il dirigea la boîte "Le Mambo " (1972-1989), chaussée de Wavre, une référence sans équivalent en Europe pour les Congolais qui résidaient en Belgique, les diplomates africains en Europe et bien sûr l'élite zaïroise $^{11}$. Chanté par de grands noms de la musique congolaise de l'époque, "Henri du Mambo " voit alors défiler les élites du pays (Zaïre), qui dépensent sans compter, souvent plusieurs dizaines de milliers de francs belges en une seule soirée.

4 Celle-ci est mobile, retirée et remise au gré des travaux entrepris par les espaces commerçants.

5 Pour une description de la vie du quartier, voir Shungu (1986) et Wamu Oyatambwe Dieudonné (2006) Matonge-Porte de Namur : un quartier africain au cour de Bruxelles ? Une étude socioculturelle, Étude réalisée pour le compte de la Commission Communautaire Française (COCOF). Le rapport n'a pas été publié à la demande des financeurs.

6 Entretiens, 2008.

7 Cf. Corijn (2004) qui avance le chiffre de $2 \%$ de résidents d'origine congolaise.

8 Partisans d'Étienne Tshisekedi, leader de I'UDPS (I'Union pour la Démocratie et le Progrès Social), historique parti d'opposition sous Mobutu, mais dont l'orientation ethnique, luba, du leader, ressort aujourd'hui fortement.

9 En référence au premier Premier ministre congolais Patrice Émery Lumumba.

10 Cf. note supra.

11 Le 27 octobre 1971, avec le décret du " recours à l'authenticité " le Congo devenait Zaïre jusqu'en 1997, date d'accession de Laurent-Désiré Kabila à la tête de l'État. 
Non sans ironie, le patron du "Mambo ", condamné à mort (par contumace) en 1978 par le président Mobutu Sese Seko pour complot contre le pouvoir ${ }^{12}$, devient alors le confident des dignitaires du régime ayant fait du "Mambo " leur établissement attitré (Demart, 2008a). Cette circulation de l'économie et des personnes est aussi intelligible à travers le trafic aérien de l'époque. La seule compagnie nationale Air-Zaïre assure cinq vols par semaine, toujours remplis de fonctionnaires, d'hommes politiques et de commerçants congolais ${ }^{13}$.

Pendant plus de cinq années, j'ai très régulièrement fréquenté le "Carrefour " à Matonge, jusqu'à en devenir une habituée, quelqu'un de la maison à qui I'on pouvait reprocher des absences prolongées ou le fait de ne pas donner de nouvelles pendant plusieurs semaines.

Suivant le " terrain ", j'étais arrivée en 2004 à Bruxelles, sur le conseil des Congolais de Toulouse auprès desquels j'avais mené des enquêtes dans les milieux associatifs et religieux : "Il faut aller à Bruxelles, c'est là qu'il y a l'élite, c'est là qu'il y a Matonge ". Effectivement I' " Histoire " était là et ce petit café donnait à voir l'intensité de dynamiques mémorielles et la singularité des rapports avec l'ancienne métropole, inaccessible depuis le pays frontalier. Outre l'aspect de contextualisation des migrations, c'était le contraste avec des milieux religieux (Demart, 2008b et 2010) appelant, au contraire, à rompre avec le passé (malheureux) à la faveur d'un avenir plein de promesses et révélé par les Écritures, qui retenait mon attention. Car au cours de ces sociabilités " matongaises ", il s'agissait bien de " faire " et de " dire " I'histoire, en dehors des circuits établis.

La mémoire du quartier Matonge, dont le patron du "Carrefour " s'avérait être un dépositaire notoire, était régulièrement ravivée par la venue d'amis, résidant à Bruxelles, Paris, Londres ou Kinshasa et de passage à Matonge. Ces retrouvailles, habituelles ou exceptionnelles, ouvraient des temporalités mémorielles fortes. Celles-ci étaient le lieu de reconstructions, auxquelles chacun apportait sa contribution, et de transmission, pour les plus jeunes ou ceux ayant connu le pouvoir de façon plus " périphérique ". La mémoire de Matonge était alors aussi celle du Congo, appréhendée par le prisme de la circulation de ses élites, politiques, économiques, artistiques ou intellectuelles et de la continuité territoriale perdurant entre le Congo et la Belgique.

L'article divisé en deux parties abordera les sociabilités " matongaises " en regard de l'évolution de la présence congolaise en Belgique, après I'Indépendance, puis les mises en forme plus organisées de l'histoire du quartier à partir des "visites guidées ". L'anthropologie sociale d'inspiration compréhensive et phénoménologique, ici mobilisée, justifiera le dévoilement progressif dans le texte, des éléments de contextualisation empirique.

\footnotetext{
12 II s'agit du procès Kalume qui a mis en cause une quarantaine d'officiers ainsi que des civils. Une quinzaine de personnes a été exécutée, accusée d'avoir fomenté un coup d'État contre le président Mobutu.

13 Elle dessert aussi Paris, Athènes, Rome, Bruxelles, Lisbonne, Cologne, Londres, etc. En outre, les liaisons Europe/Kinshasa sont aussi assurées par I'ex-Sabena (actuelle Brussels Airlines), Air France, KLM, Lufthansa, Panam, Air Afrique, Aeroflot, Ethiopian Airlines (dans les années 1980) ou encore British Airways.
} 


\section{Espace belgo-congolais : mobilités et sédentarité}

\section{Matonge : miroir des présences congolaises en Belgique}

Quartier select, le petit Matonge ou Matonge de Bruxelles se développe à la fin des années 1960, début des années 1970, autour de trois points de concentration : un centre d'accueil et d'hébergement (la "Maison africaine »), des galeries marchandes (les galeries d'Ixelles situées entre les chaussées d'Ixelles et de Wavre, et entre la chaussée de Wavre et la porte de Namur) et une boîte de nuit ("Le Mambo ") (De Clercq, 2002 ; Demart, 2008a). À cette époque, aucune immigration congolaise $n^{\prime}$ est repérable en Belgique ${ }^{14}$. L'immigration subsaharienne elle-même est embryonnaire (Kagné et Martiniello, ibid.). Les Congolais présents sont des étudiants aux côtés desquels une population très mobile se donne à voir : dignitaires du régime, diplomates, fonctionnaires et employés d'organismes publics comme les sociétés minières (la Gécamines, Kilo-Moto, la MIBA), la compagnie aérienne Air-Zaïre, les offices nationaux : de gestion du Fret maritime (I'Ogefrem) ou des transports (I'Onatra), etc. Ceux-ci viennent dans le cadre de leurs fonctions mais aussi pour " dépenser ", eu égard au très fort pouvoir d'achat dont l'élite congolaise puis zaïroise dispose. Les commerçants belges de l'époque s'en souviennent, tel ce propriétaire d'une boutique d'habits dont la seule clientèle congolaise lui rapportait jusque dans les années 1990 un chiffre d'affaires mensuel de plusieurs millions de francs belges.

Dès son origine, Matonge est donc un lieu de rencontre pour les Congolais résidant dans diverses communes bruxelloises et villes de Belgique, mais aussi d'Europe ou du Congo-Zaïre. La dynamique urbaine est congolaise mais n'épuise pas la diversité culturelle. Parmi les premiers commerçants, on trouve des Belges, des Haïtiennes, des Guinéens, un Capverdien. Dans les années 1990, c'est une véritable mosaïque qui se donnera à voir lorsque Pakistanais, Libanais, Rwandais, Sénégalais, Camerounais, ou encore Latino-américains, investiront I'espace marchand.

En attendant, et alors que Matonge se constitue comme un lieu incontournable du voyage en Europe, le statut de réfugié est impensable, synonyme de honte et de scandale. Tout un vocable traduit les stigmates associés à la demande d'asile ${ }^{15}$. Mayoyo (1995) constate que jusque dans les années 1980, il n'est pas rare d'entendre proférer l'insulte " tala ngunda oyo " (" regarde-moi ce réfugié ! "). Dans ce contexte d'intense activité circulatoire, l'installation durable n'est pas envisagée, ni envisageable, ce que rappellent inlassablement les clients du "Carrefour ": "On n'aurait jamais imaginé vivre en Europe ". Raison pour laquelle, peut-être les Congolais n'investirent pas dans l'immobilier.

La situation des étudiants se détériore toutefois rapidement. Dès 1976, les bourses délivrées par l'État zaïrois connaissent des interruptions plus ou moins brèves et en 1982, elles sont définitivement suspendues. Cette situation alimente leur contestation et leur politisation face au régime zaïrois (Bodeux et Demart, à

14 En 1961, on recense 2585 Congolais en Belgique et dix ans après 5244 (Kagné et Martiniello, 2001).

15 Le " demandeur " est désigné en lingala par le terme de ngunda, qui renvoie à la jungle ou la forêt dense, inhospitalière. La demande d'asile kobwaka nzoto, qui signifie littéralement " jeter le corps ", renvoie à l'idée du reniement. Cf. Mayoyo (1995). 
paraître), d'autant que la crise socioéconomique dans laquelle s'enfonce le pays conduit dans de nombreux cas à différer le retour.

Face au cadre législatif belge ne leur permettant pas de travailler, certains traverseront la frontière (belgo-française) ${ }^{16}$, tandis que $d^{\prime}$ autres gèreront leur précarité en travaillant illégalement ou en vivant des revenus de leurs épouses, le plus souvent assignées aux tâches domestiques. À l'encontre des tendances générales privilégiant les anciennes métropoles, la France compte à partir des années 1990, plus de Congolais que la Belgique (Lututala, 1997) qui ne voulut jamais d'une main d'œuvre (post)coloniale sur son territoire métropolitain, y compris lorsqu'elle en eut besoin.

Au début des années 1990, la sédentarisation des Congolais n'en est pas moins réelle, et relevée par l'historienne Anne Morelli (1994), récapitulant plusieurs mémoires de licence engagés sous sa direction entre 1989 et $1992^{17}$. Cette transformation de la présence congolaise, qui ne fut en définitive jamais voulue, ni par les Belges ${ }^{18}$, ni par les Congolais, est intelligible à l'échelle du quartier Matonge. En 2000, c'est une image stigmatisante qui lui est associée en regard de la délinquance juvénile qui s'y développe (De Clercq, ibid.). Les actes de violence (incivilités, délinquance) enregistrés créent une tension qui vient s'ajouter à la racialisation des rapports sociaux que l'omniprésence des contrôles policiers institue.

En 2001, des émeutes éclatent suite à une " bavure " policière. Un jeune d'origine congolaise, présumé dealer est abattu après une poursuite alors qu'il n'était pas armé. L'incident a eu lieu à la porte d'Anderlecht, mais la réaction congolaise, immédiate, a lieu à Matonge. Commerçants, clients, jeunes vont pendant quatre jours s'opposer aux forces de l'ordre. Le calme finira par se rétablir et le pouvoir communal, nouvellement élu, prendra en charge les frais relatifs au décès du jeune homme (rapatriement du corps, cérémonie funéraire, etc.). Point de départ d'une approche plus préventive, la Commune décidera de mettre en place une politique de la " main tendue " en collaborant avec les associations congolaises, créées pour prendre en charge la problématique émergente des " bandes urbaines ", mais sur une base essentiellement bénévole au contraire des politiques sociales et sécuritaires mises en place dans les années 1990 à la suite des émeutes maghrébines (Cartuyvels et Heberrecht, 2001).

La cohésion sociale se rétablira progressivement sur le territoire de Matonge, mais sans pour autant effacer les stigmates et fantasmes relatifs à l'insécurité de cet espace urbain. Pourtant, loin d'être un hors lieu dans Bruxelles ou un quartier ethnique coupé de la société globale, Matonge est aussi, dès sa constitution, fréquenté par des autochtones y compris de l'establishment. Aujourd'hui encore, il n'est pas exceptionnel d'y croiser des spécialistes belges du Congo (de

16 Outre les étudiants, on relève en France, dès 1978, la présence de quatorze « réfugiés zaïrois " (M'Bwilu, 2003).

17 Des étudiants congolais Kusukama Salabi, Tshika Yabadi, Uhala Wimbi, Mbikay Kampandha et Lusanda Ndamina.

18 L'État belge ne fit jamais appel à une main-d'œuvre (post)coloniale, du fait de la ségrégation raciale et territoriale de son dispositif colonial et du besoin de main-d'œuvre local dans la colonie. 
même que des diplomates et personnalités congolaises de passage en Europe). Cela étant, on pourrait aussi l'aborder sous I'angle d'un " espace autre ", hétérotopique (Foucault, 1967) pour l'autochtonie en regard de la population qui s'y donne à voir en certains lieux et en certaines heures : des nostalgiques (colons, missionnaires, coopérants et mercenaires au tutoiement facile, à la main leste et aux regards malsains), des personnes touchant les minimas sociaux (parce que la bière y est moins chère et que les bistrotiers congolais ont la réputation de ne jamais chasser aucun client), des indicateurs de la Sûreté de l'État et des "bobos $"^{19}$.

Actuellement, deux dynamiques tendent à s'opposer dans l'évolution territoriale et sociale du quartier Matonge : celle de la gentrification de ces quelques rues, situées entre la porte Louise et les institutions européennes, et celle d'un multiculturalisme à élaborer pour que Matonge redevienne attractif (Corijn, ibid.), à l'instar des activités culturelles $s^{\prime} y$ développant ${ }^{20}$, mais auquel les Congolais, ayant connu le " beau Matonge ", ne croient guère. C'est dans ce contexte que la cession du "Carrefour ", que le Vieux Henri et Mère Hono n'avaient pourtant repris qu'en 2003, constitue une page importante de l'histoire pour certains Congolais-e-s (de nationalité et d'origine).

\section{Temporalités et sociabilités " matongaises "}

Fin 2004, le "Carrefour " est un lieu hautement fréquenté. Le week-end, surtout, I'ambiance bat son plein. II est difficile de se trouver une place. Parmi les clients, on peut rencontrer des Congolais venant de toute la Belgique, francophone et néerlandophone, comme des Congolais résidant en RDC et de passage à Bruxelles pour missions, affaires ou vacances. Quelques mindele (Blancs) se retrouvent aussi parfois dans ces ambiances congolaises : des amoureux du Congo ou des habitués de longue date, des conjoints, des amis de clients ou du patron ou encore de simples passants attirés par la musique ou souhaitant manger africain. Sans oublier, plusieurs fois dans la soirée, la venue de vendeurs à la sauvette, ouest-africains ou asiatiques, proposant pour quelques euros des CD gravés de musique congolaise, des montres ou bijoux de contrefaçon, des jouets remuants et sonores fabriqués en Asie, etc. : "Ces gens-là, ils sont intelligents, ils travaillent pour construire au pays, pendant que nous, les Congolais, on est là, on boit, on passe notre temps à parler du pays !".

La thématique du pays occupe, en effet, une large place dans les discussions ou plus exactement dans les débats qui animent cet espace-temps communautaire. Outre le fort capital universitaire de la clientèle, c'est la musique congolaise des années 1960 et 1970 qui singularise ce café-restaurant, annonçant une clientèle d'un certain âge. Telle Mama Christine qui n'a pas son pareil pour égayer une soirée, même lorsque le moral des troupes est au plus bas. Elle aussi a connu l'émergence de Matonge, et est l'une des rares, si ce n'est l'unique, à avoir autorité sur son "frère ", Henri. À côté de leur complicité festive, c'est aussi un certain " nationalisme " qui les lie. Mama Christine est arrivée en Europe

19 Merci à Didier de Lannoy pour cette remarque judicieuse.

20 Ou du colloque Quartier Matonge, enjeux, états des lieux, perspectives, organisé par I'ASBL (association sans but lucratif) "Interface Culture ", le 12 mai 2007, Cinéma Flagey, Bruxelles. 
pour la première fois en 1960, elle avait alors seize ans. Lumumba élu, elle était en route pour Moscou avec son époux. Lumumba assassiné, les voici revenus en Belgique où ils s'installent pour que son mari puisse poursuivre ses études. Comme pour de nombreux étudiants congolais présents dès les années 1960, la Belgique est alors une extension du territoire congolais. D'ailleurs, rappellet-on, les Congolais avaient pendant la période coloniale la nationalité belge, en tant que sujets du roi. Dans cette optique, de nombreux Congolais ont dans les années 1990, et probablement au-delà, modifié leur date de naissance, en vue de faciliter la régularisation de leur statut en Belgique. Mais surtout, souligner cette modalité de présence congolaise en Europe, c'est pour certains, rappeler une " époque où I'on respectait les Congolais " malgré un racisme paradoxalement plus explicite.

Dans ce voyage dans le temps et dans l'espace auquel est invitée la chercheuse, une phrase se donne à entendre comme fil conducteur des discussions : " les Congolais n'étaient pas un peuple prédisposé à la migration ". Bien que cette affirmation annonce une histoire en marge des récits nationaux, il ne s'agira pas de mettre en perspective des mises en forme mémorielles différenciées ou divergentes, mais plutôt de s'intéresser à ce qui se donne à entendre dans les marges de la société. Car si des espaces de confrontation des mémoires voire des savoirs se donnent à voir de façon ponctuelle à Matonge et plus généralement dans le cadre de manifestations scientifiques et, ou culturelles publiques (qui pourront ensuite être discutées entre soi à Matonge), c'est plus généralement sur le mode de la contestation des discours mainstreams (populaires, médiatiques, politiques, scientifiques ou muséologiques) que des récits historiques alternatifs se construisent de façon collective et orale dans l'espacetemps de ces sociabilités.

Lorsque je n'étais pas une simple observatrice ou auditrice, c'est sur le mode de la transmission, entre témoignage et enseignement, que l'on m'a adressé ces fragments d'histoire, parfois aussi sous forme de confidence, et exceptionnellement d'invective. L'activité d'écriture qu'imposait la prise de notes au cours de cette observation participante a cependant aussi suggéré que je pouvais être une " indic" " ; ce que j'apprendrai plus tard, une fois l'affaire " réglée " entre soi.

Très vite, le journal de terrain a aussi, et surtout, symbolisé le lieu de transition que je pouvais instituer entre une histoire orale ou périphérique, et une histoire écrite, instituée ou du dehors. L'histoire orale s'élaborant au cours de ces sociabilités ${ }^{21}$ n'est toutefois pas réductible à une "culture " de l'oralité. Outre les cursus universitaires des acteurs et la lecture des quotidiens ou $\mathrm{d}^{\prime}$ hebdomadaires européens, c'est la consultation régulière pour ne pas dire quotidienne de multiples sites internet congolais (journalistiques, politiques ou religieux) qui alimente la circulation des informations au sein de la diaspora (en Belgique comme ailleurs). Certains clients sont eux-mêmes inscrits dans une dynamique d'écriture de I'histoire, passée ou en train de se faire, au travers d'initiatives associatives ou politico-médiatiques (via le Net). C'est le cas de ces trois hommes, économistes et journalistes de formation, mais n'ayant pas trouvé de débouchés dans leurs domaines respectifs. En 1999-2000, en réaction

21 Sociabilités pouvant être localisées ailleurs, dans un autre nganda ou au domicile des usagers du lieu. 
à la guerre déclenchée à l'est de la RDC par le Rwanda et I'Ouganda, ils initient un site d'information sur la RDC, en écrivant en partie sous des pseudonymes. Outre la diaspora et les milieux politiques en RDC, c'est l'attention des médias établis (en particulier CNN et BBC, qui proposèrent successivement une collaboration et des bourses pour aller se former à l'étranger) que va susciter ce média minoritaire. Perçues comme très ambivalentes, ces propositions furent refusées au titre de l'incompatibilité des lignes éditoriales. Ici, le média dit ethnique ne relève donc pas tant du registre de la représentation d'une identité collective (Rigoni, 2010), que de la " guerre de l'info " vis-à-vis des médias dominants ou impérialistes. Un système de "traçabilité " du site internet avait été mis en place afin d'identifier a minima les utilisateurs du site. Aux côtés de privés, le serveur internet pouvait ainsi relever des utilisateurs plus politiques, des ministères ou encore des services des renseignements localisés en Europe, aux États-Unis et en Israël. Des propositions politiques et financières ont aussi eu lieu, notamment de la part d'un ministère des Affaires étrangères européen, en vue de mettre un terme aux attaques "frontales " du site à l'égard de I'" Occident ". Début 2008, sur le mode du burn-out militant favorisé par la précarité des acteurs, le site a fini par cesser ses activités : " on nous créait des problèmes, du jour au lendemain, comme ça le site disparaissait, de 1999 à 2005... le site a été phagocyté trois fois de suite ", précise l'un deux.

Dans le cas associatif, on a au contraire affaire à une posture de l'entredeux favorisée par le registre non plus politique mais culturel des productions mémorielles sur le thème de l'histoire du pagne ou encore de la transmission de la mémoire par les femmes depuis la période précoloniale. Ces éclairages historiques reposent en bonne partie sur le recueil d'entretiens dont la richesse a à voir avec la trajectoire de la Présidente de l'association, Césarine Sinatu Bolya. Comme de nombreux enfants de l'élite post-coloniale, elle arrive toute jeune en Belgique au début des années 1960 et étudie dans des écoles bourgeoises de Wallonie. Mais c'est surtout l'élite congolaise (intellectuelle et artistique notamment) au sein de laquelle elle évoluera à Kinshasa comme à Bruxelles qui lui offre la possibilité de prendre en compte les récits de personnalités ayant joué un rôle à différents moments de l'histoire du Congo. Sous la forme d'expositions itinérantes ou de conférences, ces éclairages historiques obtiennent plusieurs financements de la part d'organismes locaux. Cependant, un contraste étonnant apparaît entre la richesse des données orales et leurs mises en forme et ancrage dans un support donné. Une difficulté de synthèse qui n'est pas sans induire des financements ponctuels pour ne pas dire précaires, en même temps qu'une fabuleuse source d'informations pour tout chercheur en mal de définition de son objet.

Si les échanges constitutifs des temporalités matongaises sont donc largement alimentés par d'autres dynamiques de production de l'histoire, ce type d'initiatives, aussi différencié et prégnant soit-il, ne peut toutefois être généralisé à I'ensemble des habitués du "Carrefour ". On pourrait même dire qu'il est exceptionnel. Cela étant, à côté de ce premier cercle de clients, on trouve une clientèle plus mobile et ponctuelle, sédentarisée ou non en Belgique, parfois de passage pour des raisons professionnelles, familiales ou pour des vacances, et ne pouvant faire l'économie d'un détour par Matonge. Ces clients, compatriotes, parents ou amis, s'associeront de façon momentanée à ces économies matongaises, donnant des nouvelles du pays ou de la diaspora. 


\section{Migrations, études, déqualifications et retours}

L'actualité et la politique congolaises, constamment commentées lors de ces rencontres font sans cesse retour sur le lien entre les " scandaleuses "22 richesses

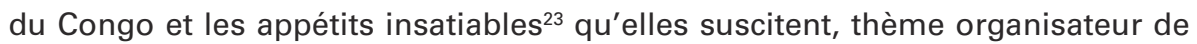
ces récits d'exil. Dans un va-et-vient constant entre la politique intérieure et la politique extérieure de la Belgique, les grands récits de l'histoire du Congo ne cessent de se mêler aux petits récits des trajectoires migratoires. Les diplômes décrochés en Europe n'ont jamais débouché sur les qualifications escomptées, ou très rarement, et généralement pour ceux arrivés tout jeunes en Belgique. On en arrive parfois au constat de la plus grande réussite sociale de ceux qui ont pris le risque de rentrer au pays, y compris lorsque le régime dictatorial de Mobutu battait son plein. Les diplômes accumulés au fil des années pour ne pas rester chômeur, et/ou pour éviter de devenir sans-papier, ne parviennent, en effet, plus à cacher la réalité de ce " temps perdu " en Europe. Et alors que des cheveux blancs commencent à être visibles chez certains, un autre discours sur la migration devient omniprésent : celui du retour.

Dans la difficulté à mettre en œuvre ce retour, l'intensité des migrations économiques vers la RDC d'autres populations, africaines et surtout asiatiques et occidentales, ne peut manquer d'être soulignée ; d'autant que l'ambassade de la RDC (située à quinze minutes à pieds du "Carrefour ") ou l'avion (" rempli de Blancs qui vont faire l'argent au Congo ") sont des lieux d'observation privilégiés de cet attrait. Une dynamique de retour n'en a pas moins lieu comme en témoigne l'effervescence circulatoire accompagnant les moments de transition politique en RDC.

En 1997, avec I'arrivée de Laurent-Désiré Kabila au pouvoir, un mouvement significatif de retour est observé, non sans conflictualités avec les Congolais de l'intérieur qui en viennent à qualifier la diaspora de " diaspourrie ", terme renvoyant à l'abandon du pays en situation de crise, au sentiment de supériorité dans les mouvements de retour et à un enrichissement rapide concomitant de l'insertion dans l'échiquier politique local, à l'instar des mobutistes. En 2006, avec les élections législatives puis présidentielles clôturant la longue période de transition politique (succédant à l'assassinat de Laurent-Désiré Kabila en 2001), de nouveaux mouvements de retour ont lieu. Tandis que des membres de la diaspora sont nommés dans le gouvernement et les entreprises publiques ou sont élus à l'Assemblée nationale ${ }^{24}$, certains tentent l'aventure, et/ou l'entreprenariat, de façon plus ou moins heureuse.

Si la (ré)insertion dans le pays d'origine est variable, la dynamique de retour n'en est pas moins effective, amorçant le plus souvent une dynamique de I'entre-deux, entre la source de revenus et le travail situés en RDC et la famille

22 Pour reprendre la célèbre expression du géologue belge Jules Cornet qualifiant dès le XIXe siècle, la province du Katanga de "scandale géologique ".

23 Une littérature très importante renvoie à ce phénomène qu'il serait impossible de restituer de façon exhaustive. Pour plus d'information, voir Braeckman (2003) et Baracyetse (2000).

24 Bien que la double nationalité ne soit pas accordée aux Congolais, un nombre significatif de députés congolais semble avoir une nationalité étrangère (occidentale ou africaine). 
installée en Belgique. Ces mobilités ne remettent pas en cause l'identification à la diaspora, à l'encontre des catégorisations scientifiques (Vertovec, 1999) à I'instar des économies familiales afférentes.

Dans un nombre significatif de cas, en effet, le projet de retour crée ou entérine, du moins dans un premier temps, des tensions conjugales, redevables de la difficulté masculine à assumer son rôle de pourvoyeur et par conséquent de " chef de ménage " en situation migratoire. Les femmes qui ont généralement eu des papiers avant leurs époux (mariage coutumier et/ou civil) semblent aussi acquérir la nationalité belge plus rapidement, ou plus facilement, tandis que les hommes qualifiés tendent non seulement à multiplier les diplômes pour éviter la déqualification et le chômage, mais mettent souvent un point d'honneur à conserver leur nationalité d'origine. Cette différenciation sexuée traduit une continuité avec les circuits d'ascension politico-sociale, essentiellement masculins, ayant prévalu jusque dans les années 1990 : obtenir un diplôme en Belgique pour intégrer de hautes fonctions en RDC. Bien que ceux-ci ne soient plus aussi efficients, ils sont largement perçus comme une voie de secours face à la faible capacité d'intégration du pays d'installation (De Keyser, Delhez et Zimmer, 2012).

Dès le jour de mon arrivée au "Carrefour ", on m'expliquait le concept colonial du " pas d'élite pas de problème " ayant abouti à un " développement parallèle " des Belges et des Congolais sur un même territoire ${ }^{25}$. L'insistance avec laquelle, la colonisation belge était, et est, analysée et disséquée, et ses grandes règles dégagées, souligne la permanence des frontières raciales et coloniales éprouvées en Belgique. Dès lors, et au regard de I'histoire longue, la multiplication des diplômes congolais peut être interprétée comme une revanche postcoloniale. Une revanche pouvant au passage brouiller les catégories autochtones lorsque dans le même temps, la catégorie " intellectuel " est appliquée à toute personne ayant étudié à I'université. L'autocritique collective n'hésite alors pas à dénoncer le "complexe de colonisé " que traduit le fait de considérer le diplôme comme une médaille ou d'accepter une position de " pupille " prédéfinie (Tshitungu, 2010) dans le champ des expertises belges ou internationales. Cela étant, le niveau d'instruction au sein de la population congolaise n'en contraste pas moins avec le non moins fort taux de chômage (Schoonvaere, 2010) au sein de ce groupe social. Une situation postcoloniale qui reste encore à investiguer et à interroger au regard d'un racisme manifestement empreint de stéréotypes coloniaux ${ }^{26}$.

\section{Géographies (post)coloniales}

\section{« Ambiance " et histoire orale}

Au cours des espaces-temps communautaires associés au "Carrefour ", les entrées et sorties des clients inscrits dans de multiples appartenances et réseaux de relations au sein de Matonge participent d'un mouvement, voire d'une forme

25 Sur l'absence de formation d'une élite intellectuelle et politique jusqu'à l'indépendance, à la faveur d'une élite morale l' "évolué " et d'un enseignement primaire généralisé, voir Ndaywel (1998).

26 Voir l'étude du centre pour l'Égalité des chances, Discrimination des personnes d'origine subsaharienne: le recyclage des stéréotypes, dossier de presse, 21 mars 2011. 
de " désordre ", que conforte, à l'intérieur du nganda, le remarquable brouhaha des voix s'imposant par-dessus la musique ou s'interpellant d'une table à l'autre. Également les éclats de rire que suscitent les blagues ou provocations de tel " amuseur ", ou les danses improvisées à l'écoute nostalgique des maîtres de la rumba congolaise Kabassele, Tabu Ley ou Franco (White, 2008). Les plus jeunes, quant à eux, feront des démonstrations très appréciées des derniers pas de danse lancés par les musiciens contemporains Papa Wemba, Koffi Olomide, Fally Ipupa ou Werrasson. Comme souvent dans les milieux congolais, la télévision est allumée mais l'espace sonore est occupé par l'ambiance du pays.

Dans cet apparent tohu bohu donc, les discussions et débats vont bon train, favorisés par l'enthousiasme des rencontres et des retrouvailles. Des tables se constituent à mesure que les clients arrivent, $n^{\prime}$ empêchant toutefois pas ces derniers de circuler d'une table à l'autre ou de dialoguer depuis des tables différentes.

L'importance des salutations annonce la dimension collective de l'usage du lieu. On ne salue pas seulement la personne que I'on connaît, mais l'ensemble des personnes présentes à sa table. Et si l'immigration tend à niveler les statuts prévalant dans le pays d'origine à la faveur du statut " d'immigré ", les modes de présentation de soi, au contraire, mettent en avant les qualifications que celles-ci se soient ou non traduites dans le champ professionnel. II est à cet égard de bon ton de ne pas demander ce que chacun fait car on sait très bien qu'ici " on se débrouille comme on peut ". Les discussions sur les difficultés de la vie en Europe, quant à elles, débouchent immanquablement sur les causes, internes et externes, de la déroute congolaise présidant à l'exil.

Dans ces débats, la récurrence de certains thèmes laisse entendre que l'histoire serait à faire. En particulier I'histoire de la colonisation-décolonisation irréductible à son cadre juridique, territorialement et historiquement défini (Mbembe, 2010). Deux niveaux se distinguent alors, tout en étant en partie imbriqués, celui du rapport colonial et/ou postcolonial pris au sens large et mobilisant des perspectives comparatives souvent très bien renseignées. Et celui du rapport particulier, belgo-congolais, qui renvoie à une histoire partagée dont fait partie I'histoire de l'immigration. Dès lors, rendre visible des modalités de présence congolaise en Europe irréductibles à l'immigration ou faire l'inventaire de ce que la Belgique " doit " au Congo participe subjectivement de la déconstruction du rapport inégalitaire éprouvé dans la migration. Dans cette mise en commun spontanée des expériences et des savoirs, I'histoire qui s'élabore ne relève pas à proprement parler de l'action collective, même si des demandes de reconnaissance et des revendications politiques sont explicitement énoncées.

Un exemple emblématique est la politique des grands travaux entreprise par le roi Léopold II avec I'argent de la colonie ${ }^{27}$ et dont les réalisations (arcade du Cinquantenaire, château de Læken, Palais de justice, etc.) sont considérées comme les marqueurs de la dette coloniale et d'une œuvre civilisatrice avant tout localisée sur le territoire de la métropole. L'idée du développement de la métropole par sa colonie s'énonce en termes de dépendance de la Belgique à l'égard du Congo. Une dépendance que cette minorité observe " en double "

27 Cette politique a été financée par la Fondation de la Couronne (spécialement créée à cet effet en 1901). 
dans les rapports diplomatiques entre les deux pays ou dans les " mises en scène " et "fabriques " d'une dépendance qui serait celle de la RDC à l'égard de la Belgique ${ }^{28}$ et plus globalement de l'Occident vis-à-vis de l'Afrique.

Un autre registre est celui du pouvoir économique des Congolais qui, au lendemain de l'indépendance, nivela pour ne pas dire inversa, la hiérarchisation sociale, sur base raciale, ayant prévalu pendant la colonisation, léopoldienne (1885-1908) puis belge (1908-1960). Ces récits alternatifs de I'histoire belgocongolaise peuvent faire référence à l'assiduité avec laquelle des notables belges et français assistaient aux festivités qu'organisait Mobutu dans sa villa du Cap Martin (Roquebrune), à la générosité de ses " cadeaux " ou au fabuleux pouvoir d'achat des mobutistes, dont l'immobilier reste un marqueur incontournable. Durant les décennies 1970 et 1980, de nombreux dignitaires du régime achetèrent toujours cash, des villas en Europe : en France, en Belgique et dans une moindre mesure en Suisse (la villa de Mobutu est située au bord du lac Léman) et en Allemagne (Brême et Munich). En 1997, avec le changement de régime (et leur sortie des sphères du pouvoir), ces notables n'ont globalement plus été en mesure d'honorer leurs créances ; les biens immobiliers ont alors été dans leur très grande majorité revendus aux enchères. Ces villas font aujourd'hui partie des marqueurs territoriaux renseignant I'histoire de la présence congolaise en Belgique aux côtés des constructions financées par l'argent de la colonie et qui, a contrario, rappellent I'histoire de la présence belge au Congo.

Cette double référence territoriale traverse, pour ne pas dire structure, l'histoire de la migration indissociable des rapports belgo-congolais, dont l'objectivation amène à visibilité la chronologie politique congolaise : I'assassinat de Lumumba (1961), premier Premier ministre élu (de Witte, 2000 ; Omasombo, 2004), le " placement " de Mobutu Sese Seko à la tête de l'État congolais (19651997), I'assassinat du président Laurent-Désiré Kabila (2001) qui I'avait évincé (1997) et, plus récemment, l'agression ougando-rwandaise (et dans une moindre mesure burundaise) à l'est de la RDC. Ces références permanentes à des cadres macrosociaux et historiques, qu'il serait trop long de développer en détail, renvoient à une histoire dont la mise en forme ne fait toujours pas consensus avec la société globale. En particulier, en ce qui concerne les modes de participation des puissances étrangères (dont l'ancienne puissance coloniale, aujourd'hui " société d'accueil "), à l'institution ou à la destitution des pouvoirs qui se sont succédé au Congo (Bodeux et Demart, ibid.). Néanmoins, si au niveau du "Carrefour ", I'imbrication des géographies congolaises à Bruxelles et des temporalités belges en RDC est mise en perspective de façon informelle et entre soi, elle peut ailleurs faire l'objet de transmissions beaucoup plus structurées, y compris à l'initiative d'acteurs extérieurs aux milieux congolais.

28 II est à cet égard intéressant de souligner l'évolution du lexique pour désigner ces rapports belgo-congolais : depuis celui de neveu à oncle à celui de cousins. Si pendant longtemps, les Belges étaient les "noko " (les oncles), la volonté de transformer ce rapport dans le sens de rapports plus égalitaires et fraternels s'est faite entendre à différentes reprises autour du Cinquantenaire et après. Ainsi, lors du vernissage de I'exposition d'Antoine Tshitungu sur Paul Panda Fernana à I'espace culturel Senghor de la commune d'Etterbeek (Bruxelles), I'Ambassadeur de RDC soulignait que les rapports belgo-congolais étaient et devaient désormais être ceux de " cousins ", autrement dit d'une fratrie relevant de la parenté tout à la fois proche et distante (car il ne s'agit pas des cousins au sens occidental du terme qui ici sera repris par la catégorie générique de " frères " et " sœurs ") et donc dans la perspective de rapports plus égalitaires. 


\section{Visites à Matonge, interculturalité et liens (post)coloniaux}

Depuis les années 2000, il n'est pas rare de voir des groupes visiter le quartier Matonge en compagnie d'un guide. Aucune histoire " officielle " n'est cependant établie et de grandes disparités existent selon que ces visites sont organisées par des associations congolaises comme l'Observatoire BaYaya, par l'érudit écrivain Antoine Tshitungu, ou par des offices de tourisme qui seraient au nombre d'une quinzaine.

Pour I'Observatoire Ba Yaya, intervenant dans le champ de la jeunesse belgosubsaharienne et mobilisé dans les années 2000 par les pouvoirs communaux pour rétablir le lien social dans Matonge (cf. supra), la visite est avant tout I'occasion de déconstruire les préjugés liés à l' "immigration africaine ", comme I'explique le fondateur de I'association, Ngyess Lazalo Ndoma (entretien, 2011) :

"À l'époque, on vivait avec ces populations différentes, Africains, Belges, il y avait des habitués qui étaient devenus des Africains en fait, on ne voyait même plus la différence, mais à partir des années 2000, le quartier est devenu difficile, y'avait une crise... c'est vrai nos habitués étaient toujours là mais en plus des gens qui venaient pour voir. Ce regard-là était nouveau, certains prenaient des photos, y'avaient des caméras qui envoyaient des images négatives. Pour les commerçants, c'est comme s'ils étaient un zoo [...] ".

C'est alors que l'Observatoire décide d'élargir ses fonctions de médiation sociale en mettant en place des visites guidées du quartier, une à plusieurs fois par mois selon la demande et surtout selon les disponibilités (la moitié des demandes est refusée). Celles-ci peuvent faire suite à une demande de la Commune, d'écoles (primaires et secondaires), d'offices de tourisme, de politiciens, d'universités ou de hautes écoles (généralement les départements ou cursus sociaux), ou encore d'ONG " qui pensent que c'est un préalable pour aller en Afrique, alors que ce serait plutôt le contraire " (Mireille-Tsheusi Robert, Présidente de l'association, entretien, 2011) et enfin par le bouche à oreille. La majorité du public est belge dont $60 \%$ vient de la " province profonde ", essentiellement flamande. Bien que le public ait déjà entendu parler de Matonge à la télévision, les questions concernant le quartier sont limitées :

"Qu'est-ce que ça veut dire Matonge ? Et encore y'en a qui pensent même pas à demander ce que ça veut dire. Et depuis, quand le quartier a-t-il été créé ? Et qui l'a créé ? Et aussi ça se limite où ?" (ibid.).

Par contre un certain nombre de questions vont porter sur les Noirs, l'immigration africaine et la différence culturelle :

"Ce qui m'intéresse dans cette visite c'est la déconstruction de ce racisme primaire.

On a l'impression que ça n'existe plus, sauf qu'en fait les gens n'osent pas en parler et ils partent dans un racisme secondaire à défaut de pouvoir... on a des questions mais très bêtes : je peux toucher vos cheveux? Je peux toucher votre peau? Est-ce que vous mettez quelque chose en plus pour que ce soit si foncé, parce que c'est foncé quand même ? Mais vous parlez bien français quand même, "mais j'ai grandi ici", c'est comme si en fait c'était quelque chose de physique qui était inscrit en nous, même si tu naissais en Chine tu pourras jamais parler bien le chinois " (ibid.). 
À un autre niveau, ce sera la vie quotidienne de cette minorité belge, associée ou non au quartier, qui fera l'objet de questions :

"Comment on fait les cheveux? Est-ce que ça reste à vie ou ça part? Qu'est-ce que vous mangez? Est-ce que vous mangez ça tout le temps ou vous mangez aussi des frites de temps en temps? Est-ce que y'a que des Africains ici ou est-ce que c'est mélangé? Est-ce que les gens habitent ici ? Est-ce que parmi les Africains, $y^{\prime}$ a plusieurs nationalités ou que les Congolais? Est-ce que vous vous entendez bien avec les Maghrébins? On n'en voit pas dans le quartier, est-ce que vous êtes en guerre ? Est-ce que c'est vous qui contrôlez le quartier? Ou c'est quand même la Commune qui contrôle le quartier [rires]?

$Y^{\prime}$ a combien d'Africains en Belgique? Depuis quand êtes-vous là ? Pourquoi vous ne repartez pas? Est-ce que tous les jeunes sont violents? Pourquoi est-ce que vous rentrez pas les poubelles? Pourquoi est-ce qu'on parle de Dieu tout le temps? [...] Est-ce que c'est vrai que tous les Congolais sont des sapeurs? Mais parfois c'est "vous être quand même très coquets, hein ? Vous dépensez beaucoup d'argent pour la coquetterie, hein, regardez-vous vous êtes coiffés et tout !" Moi je dis, "oui, vous ne dépensez pas d'argent quand vous allez chez le coiffeur [rires] ? Ça vous coûte combien ? Ben moi c'est le même prix [rires]" [...]" (ibid.).

Il apparaît alors que si pour les guides, la visite est « clairement un prétexte à l'interculturalité ", il en va de même pour le public au travers de questions " qu'ils n'osent pas poser donc c'est bien qu'ils aient un cadre pour le faire, et qu'ils soient assez en confiance pour le faire [...] d'un point de vue interculturel, c'est génial de faire ça " (ibid.).

En outre, ces visites permettent aussi aux commerçants d'être en confiance, ce qui n'est pas le cas avec les organismes touristiques (organisant quasiment chaque jour des visites) :

"Les commerçants n'aiment pas, se sentent comme dans un zoo, ce sont des guides européens, j'ai l'impression qu'ils sont surtout sur des représentations... les quelques visites que j'ai suivies étaient très décevantes " (ibid.).

C'est également I'avis d'Antoine Tshitungu, docteur en littérature, ayant entrepris depuis des années un vaste travail de recherche sur I'histoire coloniale. Lui aussi organise des visites à la demande mais pour ceux " qui veulent avoir une idée saine du quartier". C'est généralement suite à ses écrits et interventions dans les médias, radio et télévisés qu'il est sollicité. Il a ainsi été contacté par la télévision allemande ZDF dans le cadre du Cinquantenaire de I'Indépendance du Congo pour effectuer une visite de l'ensemble des monuments historiques liés à l'histoire coloniale à Bruxelles. Depuis cette émission, il reçoit plusieurs demandes d'Allemands venant en Belgique pour tourisme. Le public peut également venir d'autres pays comme la Suisse ou plus improbable, la Nouvelle-Calédonie. Une association I'a ainsi contacté pour des jeunes de Nouméa "en situation difficile ". Étrangement, "ils comprennent tout. Quand on est arrivé devant la statue de Léopold II, y'avait pas besoin d'explications sophistiquées, ils avaient tout compris, ils savent ce que c'est la colonisation" (Antoine Tshitungu, entretien, 2011). 
Les agences de tourisme se sont également tournées vers lui, mais :

"Moi je voulais former les gens et eux ne voulaient pas, donc ces demandes sont restées lettre morte car en aucun cas je ne peux avaliser les discours que j'entends dans la bouche des guides. Et puis visiter un quartier comme ça, ça doit se faire avec le respect des gens " (ibid.).

Ici donc, la visite est explicitement historique et articulée aux " traces de la colonisation ", à ses différentes périodes et aux marqueurs de l'histoire belgocongolaise. Les frontières du quartier sont bien plus larges et poreuses que celles de l'actuel Matonge. On retrouve toujours le triangle, chaussée de Wavre, chaussée d'Ixelles et Porte de Namur, mais dans un périmètre englobant la place du Trône, la place royale et leurs alentours. Cette cartographie amène à visibilité une profondeur historique des lieux et parfois, d'étonnantes reconversions comme le montre le Bruxellois Lucas Catherine dans son livre passionnant, Promenade au Congo $(2010)^{29}$, sur lequel nous allons largement nous appuyer.

\section{Enchevêtrements postcoloniaux et traces coloniales dans l'espace de la ville}

Les visites organisées par Antoine Tshitungu ou Lucas Catherine montrent qu'en définitive, Matonge s'est développé à proximité, voire dans les balises du quartier colonial, non pas en raison d'une ouverture particulière mais d'une présence congolaise dans la ville. Une présence qui est celle des coloniaux dont les lieux de loisirs, où se mêleront dans les années 1950 les premiers Africains ${ }^{30}$, jouxtent les institutions coloniales : le ministère des Colonies situé place Royale (où siège l'actuelle cour constitutionnelle) et les bureaux des institutions et des sociétés coloniales.

Dans les années 1950, la rue Stassart, adjacente à la porte de Namur, concentre les cafés que fréquentent les coloniaux, dont les boîtes jouant du jazz, musique nègre par excellence (Antoine Tshitungu, entretien, 2011). La direction de ces boîtes " blacks ", d'abord tenues par des Antillais, de nationalité française, se diversifie à la fin des années 1950. Le "Black and White " sera tenu par un Belge, "les Anges Noirs ", par un Capverdien sénégalais et "le Baninga " par deux métis congolais. Mobutu, alors étudiant stagiaire en journalisme, le fréquente avec assiduité. Parmi les cafés "congolais", on trouve aussi le club de "l'union des femmes coloniales " ${ }^{31}$, préparant les femmes à être de bonnes épouses en colonie.

Des conférences sont également organisées dans ce quartier qui est, en définitive celui du "lobby colonial " et d'un " cercle africain " (ibid.) dont les traces sont encore aujourd'hui intelligibles au travers des associations d'anciens coloniaux domiciliées dans cette rue.

\footnotetext{
29 Comme Antoine Tshitungu, son questionnement dépasse Matonge à la faveur de I'histoire coloniale dans Bruxelles (les espaces verts, le Cinquantenaire, Tervuren, le monument Edmond Thieffry à Etterbeek, etc.) et en Belgique (Anvers, Ostende), mais se concentre surtout sur la période léopoldienne de la colonisation. Bien que ses visites de Matonge soient ponctuelles et circonstancielles, il a mis son livre à la disposition d'organismes souhaitant effectuer de façon plus systématique les visites du quartier.

30 Sur la présence des Congolais en métropole, voir Cornet (2004) et Kagné (2001).

31 Sur les coloniaux en Belgique, voir Gillet (2008).
} 
Dans les années 1960, la présence congolaise en Belgique s'intensifie avec la venue d'étudiants. Monique van der Straten Waillet, dont le frère est missionnaire en Afrique, crée "la Maison africaine " à leur destination. En 1969, ce foyer s'installe rue Alsace Lorraine de l'autre côté de la chaussée d'Ixelles. Pour une somme modique, la Maisaf offre le gîte et le couvert en même temps qu'un espace social et culturel unique qui favorise l'organisation de conférences thématiques et de fêtes, en même temps que la venue d'orchestres congolais. Dans les années 1970, les Maisons africaines de Charleroi, Liège et Anvers ferment à cause de restrictions budgétaires de l'État. Seule la Maisaf de Bruxelles reste ouverte, rachetée, nous confie l'ancien gérant de la Maisaf liégeoise (Henri Hockins, qui fut ensuite patron du "Mambo " puis du "Carrefour "), par les présidents Mobutu (République démocratique du Congo, ex-Zaïre) et Houphouët Boigny (Côte d'Ivoire) qui en feront don à la directrice. Elle est aujourd'hui subventionnée par la Direction générale de la coopération au développement (DGCD). Le conseil d'administration est essentiellement " belgo-belge ".

À quelques pas de là, Chaussée deWavre, se dessinent, dès 1968, les prémices de "Matonge ", avec la reprise de la boîte de nuit " la Marie Galante " - située chaussée de Wavre - par un ancien employé des "Anges Noirs ", Kat Kadio. En 1970, il remet son commerce au Guinéen Conté Mamaduba, alors portier à Cocodi. Ce dernier le rebaptise "Le Mambo ", les employés sont exclusivement congolais. En 1972, décidé à retourner en Guinée Conakry, il remet "Le Mambo " à Henri Hockins, alors un de ses employés (et dont la bourse accordée par l'État congolais n'est plus payée) et à son épouse belge Madeleine D'Hoerane.

Deux, trois années plus tard, un autre point de concentration des Congolais se dessine, entre les chaussées de Wavre et d'lxelles. II s'agit des galeries chics d'Ixelles, progressivement désinvesties par les commerçants belges à la faveur des galeries de la Toison d'or, récemment inaugurées dans le quartier Louise. C'est alors que Papa Jean (Lubanda), dont le beau-père (Jean Médard) avait ouvert dans la galerie un magasin de pagnes peu rentable, transforme le commerce en un petit café, le "nganda Matonge ". On y trouve de la bière congolaise, la Primus ou la Skol, ainsi qu'une petite restauration typique. $\mathrm{D}^{\prime}$ autres commerces et ngandas viendront composer ce nouveau paysage commercial dans les galeries mais également dans les rues avoisinantes, bien au-delà de l'actuelle délimitation de Matonge. "Matonge " était né32.

Les visites historiques de Matonge sont loin de s'arrêter à cette période somme toute récente dans I'histoire du quartier qui, resitué dans l'axe porte de Namur, Trône et place royale, où les institutions et les sociétés coloniales prirent place dès les années 1880, donne à voir l'entièreté de l'histoire coloniale. Et dans ses réagencements, les divers processus de colonisation et de décolonisation. Comme le souligne astucieusement Catherine, le roi Léopold II qui concrétisait dans les années 1880 son rêve d'acquérir un empire colonial, dans lequel il ne mit jamais les pieds, " n'avait qu'à sortir de son jardin pour gérer son Congo" (Lucas Catherine, entretien, 2011).

32 D'autres sources mentionnent l'inauguration de la Maison Africaine en 1970 en présence de la reine Fabiola, au cours de laquelle I'un des commerçants, congolais, papa Dialot, aurait qualifié cette nouvelle dynamique urbaine de "Matonge ". Mais celle-ci n'a pas été confirmée au cours de nos enquêtes. 
La colonisation du Congo belge (son acquisition, le tracé de ses frontières et la fine négociation de sa possession auprès des puissances de l'époque) on le sait, fut d'abord l'entreprise d'un seul homme, le jeune roi Léopold II qui, contre l'avis général de la classe politique de l'époque, batailla pour la possession et l'exploitation de ce vaste territoire (Emerson, 1980). C'est ainsi que la première période coloniale, léopoldienne (1885-1908) s'organisa autour de l'espace royal.

Les visites commencent souvent à proximité du Palais royal, place du trône, où se dresse la statue de Léopold II à l'arrière de laquelle on peut encore lire que "le cuivre et l'étain de cette statue proviennent du Congo belge. Ils ont été fournis gracieusement par I'union minière du Haut Katanga ". Face à elle se trouve la banque ING qui fut autrefois le siège de la banque Lambert, la section locale des Rothschild.

Amis intimes de la famille royale, les banquiers Rothschild gérèrent la fortune personnelle du roi comme le budget de l'État, en partenariat avec la Société Générale qui trente ans plus tard devait contrôler près des trois quarts de l'économie congolaise (Catherine, 2010 ; Ndaywel, 1998 : 390). C'est d'ailleurs, le baron Lambert membre de I'" Association internationale du Congo " et beau-fils des Rothschild, qui finança le "Comité d'études du Haut Congo " fondé en 1878, lui-même destiné à financer les premières expéditions au Congo, avant son acquisition. II devint par la suite I'administrateur de la Compagnie du Katanga et le fondateur de la banque du Congo qui deviendra plus tard la Belgolaise (avant de fusionner en 2000 avec Fortis).

Depuis la place royale, on peut emprunter la rue de Brederode, puis la rue de Namur, lesquelles pourraient à elles seules résumer la politique coloniale du roi Léopold II. Réputée être le centre du pouvoir colonial vers 1900, la rue Brederode, rattachée aux arrières du Palais royal, fut tout à la fois le quartier général du "Congo indépendant ", de la société "les amis et serviteurs de Léopold II " (fondée en 1930, après la mort du roi et qui devint en 1950 les " admirateurs de Léopold II ") et de différentes sociétés coloniales. Aujourd'hui, on y trouve la Fondation du roi Baudouin.

D'autres sociétés de I' "État indépendant du Congo " (appellation par laquelle était désigné le Congo de Léopold II) se trouvaient à proximité, dans la rue Thérésienne perpendiculaire, notamment les cimenteries du Katanga (Cimenkat) ou la société d'entreprises de travaux en béton au Katanga (Trabekat).

L'extrémité de la rue Brederode est également perpendiculaire à la rue de Namur dans laquelle se trouve jusqu'aujourd'hui un bâtiment de style néoclassique dont la plaque mentionne l'existence, autrefois, d'une "École militaire ". L'histoire alternative, nous apprend que dès 1876, cet édifice fut le quartier général de l' "Association Internationale Africaine " (à partir de laquelle Léopold II promut son projet colonial en tant qu'œuvre humanitaire), puis le "Comité d'études du Haut Congo " (qui finança les explorations de Stanley) en 1878 et dans les années 1930 le " service d'Hygiène du ministère des Colonies ". En face de ce bâtiment siégeait la " banque belge d'Afrique ", un peu plus loin, I'hôtel Barbanson où se trouvaient les bureaux de plusieurs administrations coloniales et enfin la "Banque centrale du Congo belge et du Ruanda Urundi " (dont la Belgique prit la tutelle au sortir de la Première Guerre mondiale). 
Un peu plus haut dans la rue se trouvaient des compagnies industrielles de transport au Stanley-Pool (CITAS) et de produits chimiques et pharmaceutiques du Congo (COPHAC). Enfin, la rue des petites Carmes, également perpendiculaire à la rue de Namur, hébergeait le "Comité spécial du Katanga " et la "Société pour la colonisation belge au Katanga " où se trouve aujourd'hui le ministère des Affaires étrangères.

Au coin de la place du Trône et de la rue Ducale se trouvaient les «Écuries de la Reine " et entre 1890 et 1897, les services administratifs du Congo indépendant (après avoir été rue Brederode et avant d'être déplacés à l'hôtel Brabanson). On pourrait aussi citer, entre la place du Trône et la rue Brederode, la rue de la Pépinière où se tenait le bureau de presse associé à la campagne " pro Congo de Léopold II " lors de ladite "Campagne anti-Congo " qui conduisit à la reprise du Congo par la Belgique en 1908.

Outre la remise en cause de la liberté de commerce promise aux grandes puissances lors de la conférence de Berlin, c'est l'œuvre humanitaire (contre la " traite des Arabes ") du projet colonial, ayant favorisé I'octroi de cet immense territoire à la " petite Belgique ", qui fut violemment remise en cause. Alors qu'au début du siècle, le roi était enfin assuré du succès économique de son entreprise (qui fut un véritable gouffre financier, dans un premier temps), il se voit éclaboussé par un scandale international sans précédent suite à la dénonciation anglo-saxonne des pratiques destinées à accroître la production du caoutchouc (" mains coupées ", expéditions punitives, prise d'otages, etc.). Contraint, il remet " son " Congo à l'État belge.

S'ouvre alors la seconde période coloniale dont le centre économique se déploie autour du parc royal avec dans la rue Montoyer, les savonneries du Congo, la société anversoise des entreprises commerciales du Congo ou encore les Huileries du Congo. La rue Ducale, à proximité, abrite plusieurs entreprises coloniales (Eterco, Eternit du Congo, etc.) tandis que de l'autre côté du parc royal, dans la rue royale et ses rues latérales, s'installent la Société Générale, I'Union minière du Haut-Katanga ${ }^{33}$ (uranium) et la Forminière (exploitation du bois précieux et des mines, notamment de diamant). Principal actionnaire de ces deux sociétés : la Société Générale qui, en 1928, contrôle $72 \%$ de l'économie congolaise (Catherine, 2010). Dans la rue latérale, rue Montagne du Parc, se trouvent la société coloniale des mines, les chemins de fer du bas Congo et du Katanga et la compagnie foncière du Katanga (COFOKA). C'est enfin les services administratifs du ministère des Colonies (bibliothèque africaine, archives des colonies, etc.) qui, à partir de 1925, s'installeront à côté de l'Église de Saint-Jean de Coudenberg, place royale.

Le déplacement des associations de coloniaux est également emblématique puisqu'à l'angle de la rue de Namur et de la place royale se trouvait en 1889 " le cercle Africain ", la première association de coloniaux qui devint en 1912, "le cercle de l'union royale coloniale belge " avant de déménager dans la fameuse rue de Stassart. À l'époque, le carrefour de la porte de Namur et le quartier admi-

33 Le Congo était alors le seul pays fournisseur d'uranium dont la Société Générale détenait le monopole ; c'est d'ailleurs l'uranium des mines de Shinkolobwe (Katanga) qui fut utilisé pour les premières bombes atomiques qui furent larguées sur Hiroshima et Nagasaki. 
nistratif de la rue de Brederode ne sont pas séparés par la " petite ceinture " ( ring) qui fut construite en 1958, à l'occasion de l'exposition universelle, donnant pour la première fois aux Congolais l'opportunité de découvrir collectivement la métropole (et d'avoir des contacts avec le monde politique et syndical belge, également littéraire et panafricain). Jusque-là, cet espace n'était que verdure (Lucas Catherine, entretien 2011).

\section{Conclusion}

Cette ethnographie de Matonge est très loin d'épuiser, d'une part la diversité sociale du quartier (Shungu, ibid. ; Oyatambwe, ibid.), d'autre part, les dynamiques mémorielles à l'œuvre. Bien que le "Carrefour " soit tout autant fréquenté par des hommes que par des femmes, la variable genre, comme nous le faisait remarquer Antoine Tshitungu, pourrait bien différencier la mémoire associée au quartier et la forme des revendications politiques ${ }^{34}$.

Toutefois, le contraste entre l'intensité de ces dynamiques mémorielles et l'absence d'intérêt de ce public pour les institutions à vocation interculturelle ou pour le projet de modernisation du musée de Tervuren ${ }^{35}$, est significatif. On peut au passage se demander si la volonté de transformation du musée colonial en musée postcolonial, capable de produire une autocritique affranchie de toute logique d'accumulation compulsive ${ }^{36}$, peut s'opérer en dehors de l'identité assumée de l'institution en tant que lieu de commémoration. Cela étant, le " développement parallèle " que ces circuits périphériques d'élaboration et de transmission de l'histoire, donnent à voir, ne signifie pas que les modes de transmission de ces récits divergents soient organisés, homogènes ou même parfaitement étanches. À l'écoute des responsables associatifs, il apparaît même que la transmission intergénérationnelle (de l'histoire coloniale et migratoire) ne s'opère pas.

Or, dans un contexte où les soixante-quinze années d'histoire coloniale ne font pas véritablement partie du programme scolaire obligatoire, et où le débat postcolonial investit difficilement l'espace public, la mise en visibilité des lieux et de leur histoire pourrait fournir une véritable alternative, non sans souligner le caractère dérisoire des négociations, pourtant hautement symboliques, engagées depuis des années à Bruxelles entre militants et politiques en vue du retrait de " la rue des Colonies " et de l'avènement d'une " rue Lumumba " ${ }^{37}$. Sachant par ailleurs, qu'un certain nombre de rues, notamment dans la Commune d'Etter-

34 Sur la participation politique des femmes concernant le pays d'origine, voir Chideka et Godin (2010).

35 Le Musée royal d'Afrique centrale, anciennement Musée du Congo (1898) et Musée du Congo belge (1908).

36 Cf. Communication de Bambi Ceuppens lors de la Rencontre internationale « Sujet/ Objet migrants ", 13-14 octobre 2011, Musée royal de I'Afrique centrale, Tervuren. Sur le processus de modernisation du Musée, voir aussi Bragard (2011) et pour une critique virulente, Rahier (2003).

37 Alors que l'État belge (ayant reconnu l'implication du gouvernement belge de l'époque dans I'assassinat du premier Premier ministre congolais) s'était engagé à financer une "Fondation Lumumba " (qui vit le jour en 2002), celle-ci n'aurait jusqu'à présent reçu aucun subside. 
beek limitrophe d'Ixelles, porte des noms de coloniaux ${ }^{38}$ et que les marqueurs territoriaux de I'histoire (post)coloniale peuvent être circonstanciés. C'est par exemple, le cas de ce petit bureau qui, au cours des années 1960, se souviennent certains Congolais et Belges, recrutait dans le quartier Matonge des volontaires et des mercenaires pour aller au Katanga, dont la sécession, soutenue par l'État belge, allait comme on le sait, fortement remettre en cause (onze jours après I'Indépendance du Congo) les conditions politiquement négociées du principe de souveraineté du jeune État.

Autrement dit, le différentiel des mémoires (post)coloniales que ce " développement parallèle " suggère, contraste fortement avec la logique postcoloniale de l'enchevêtrement (Mbembe, ibid.) qu'amène à visibilité I'histoire orale à Matonge à l'instar de l'extraordinaire imbrication des géographies congolaises en Belgique et des temporalités belges en RDC dont procède en définitive ce quartier congolais.

\section{Références bibliographiques}

Baracyeste Pierre (2000) L'enjeu géopolitique des sociétés minières internationales en République Démocratique du Congo (ex-Zaïre), L'Observatoire de I'Afrique centrale, 3 (35), [en ligne]. URL : http://membres.multimania.fr/obsac/ OBSN3V35-Baracyetse.html

Bodeux Leila and Demart Sarah (forthcoming, 2013) Postcolonial stakes of DRC political space: 50 years after independence, African Diaspora.

Braeckman Colette (2003) Les nouveaux prédateurs : politique des puissances en Afrique centrale, Paris, Fayard, 305 p.

Bragard Véronique (2011) 'Indépendance!': The Belgo-Congolese Dispute in the Tervuren Museum, Human Architecture, Journal of the Sociology of SelfKnowledge, 9 (4), pp. 93-104.

Cartuyvels Yves et Heberecht Patrick (2001) La politique fédérale belge de sécurité et de prévention de la criminalité, Déviance et société, 25 (4), pp. 403-426.

Catherine Lucas (2010) Promenade au Congo. Petit guide anticolonial de Belgique, Aden, 176 p.

Corijn Éric (2004) Matongé, centre multiculturel à Bruxelles, Politique, 35, pp. 32-33.

Cornet Anne (2004) Les Congolais en Belgique aux XIXe et XXe siècles, in Anne Morelli Éd., Histoire des étrangers et de l'immigration en Belgique de la préhistoire à nos jours, Bruxelles, Couleurs Livres, pp. 375-400.

Chideka Mado et Godin Marie (2010) L'activisme des femmes congolaises en RDC et en Belgique, Revue Migrations forcées, 36 [en ligne]. URL : http://www. fmreview.org/fr/RDCongo/godin\%20chideka.htm

De Clercq Dieter (2002) Everyday urban space in Matonge, OASE Journal for architecture, 54, pp. 62-85.

38 Camille Coquilhat, Baron Dhanis, Général Henry, Baron Lambert, autant de noms associés à l'aventure coloniale et à ses prétentions civilisatrices; et dont la liste est loin d'être exhaustive. 
Demart Sarah (2010) Les territoires de la délivrance. Mises en perspective historique et plurilocalisée du réveil congolais (Bruxelles, Kinshasa, Paris, Toulouse, thèse en co-tutelle, Université Louvain-la-Neuve/Université Toulouse-le-Mirail, $616 \mathrm{p}$.

Demart Sarah (2008a), De la distinction au stigmate, Matonge, un quartier congolais dans Bruxelles, Les Cahiers de la fonderie, 38, pp. 58-62.

Demart Sarah (2008b) Le " combat pour l'intégration " des églises issues du Réveil congolais (RDC), Revue Européenne des Migrations Internationales, 24 (3), pp. 145-167.

De Keyser Thomas, Delhez Philippe et Zimmer Hélène (2012) L'insertion des personnes d'origine étrangère sur le marché du travail, $B N B$, revue économique, pp. 25-44.

De Witte Ludo (2000) L'assassinat de Lumumba, Paris, Karthala, 407 p.

Emerson Barbara (1980) Léopold II, le royaume et l'empire, Duculot, 324 p.

Gillet Florence (2008) Congo rêvé ? Congo détruit... Les anciens coloniaux belges aux prises avec une société en repentir. Enquête sur la face émergée d'une mémoire, CHTP-BEG, 19, pp. 79-133.

Jewsiewicki Bogumil (2003) Mami Wata, la peinture urbaine au Congo, Paris, Gallimard, 236 p.

Kagné Bonaventure et Martiniello Marco (2001) L'immigration subsaharienne en Belgique, Courrier hebdomadaire, CRIPS, 1721, 50 p.

Kagné Bonaventure (2001) L'immigration d'origine subsaharienne avant 1960 : la Belgique découvre "l'Africain ", Courrier Hebdomadaire, CRISP, 1721, Complément d'août 2001, 15 p.

Lututala Bernard (1997) L'élargissement de l'espace de vie des Africains : comment le pays des " oncles " européens devient aussi celui des "neveux " africains, RevueTiers-Monde, 150, pp. 333-346.

Mayoyo Bitumba Tipo-Tipo (1995) Migration Sud/Nord : levier ou obstacle ? Les Zaïrois en Belgique, Cahiers africains, 13.

Mbembe Achille (2010) Sortir de la grande nuit. Essai sur l'Afrique décolonisée, Paris, La Découverte, 243 p.

M'bwilu Gabriel (2003) L'intégration des immigrants zaïrois en France : 19801994, Atelier national de reproduction des thèses, 338 p.

Morelli Anne (1994) Les Zaïrois en Belgique sont-ils des immigrés ?, Cahiers africains, 9-10-11, pp. 152-154.

Ndaywel ê Nziem Isidore (1998) Histoire générale du Congo. De l'héritage ancien à la République Démocratique, Paris/Bruxelles, De Boeck \& Larcier, 955 p.

Omasombo Tshonda (2004) Lumumba, drame sans fin et deuil inachevé de la colonisation, Cahiers d'études africaines, 173-174, pp. 221-226.

Rahier Jean Muteba (2003) The Ghost of Leopold II: The Belgian Royal Museum of Central Africa and Its Dusty Colonialist Exhibition, Research in African Literatures, 34 (1), pp. 58-83. 
Rigoni Isabelle (2010) Éditorial. Les médias des minorités ethniques. Représenter l'identité collective sur la scène publique, Revue Européenne des Migrations Internationales, 26 (1), pp. 7-16.

Schoonvaere Quentin (2009) Étude de la migration congolaise et de son impact sur la présence congolaise en Belgique : analyse des principales données démographiques, Mémoire présenté dans le cadre du Master en Sciences de la Population et du Développement, université catholique de Louvain-la-Neuve, $128 \mathrm{p}$.

Shungu Ekanga (1986) L'Afrique noire à Bruxelles, Bruxelles, Les éditions européennes, $174 \mathrm{p}$.

Tshitungu Antoine (2010) Belgique : une mémoire coloniale sélective, Politis, 65, pp. 36-38.

Vertovec Steven (1999) Three meaning of "diaspora", exemplified by South Asian religions, Diaspora, 6 (3), pp. 277-300.

White Bob (2008) Rumba Rules: The Politics of Dance Music in Mobutu's Zaire, Duke University Press, 328 p. 


\section{Sarah Demart}

\section{Histoire orale à Matonge (Bruxelles) : un miroir postcolonial}

Cet article explore les mises en forme mémorielles associées à un quartier urbain. Naguère "select ", le quartier Matonge à Bruxelles s'est développé à la fin des années 1960, début des années 1970, à un moment où aucune immigration congolaise n'était repérable en Belgique. Aujourd'hui en phase de devenir " africain " ou " multiculturel ", Matonge reste un lieu incontournable des rencontres et des festivités congolaises au cours desquelles une " autre " histoire se donne à entendre. À partir d'une approche ethnographique inscrite dans la durée, nous avons interrogé I'histoire de ce quartier dont les marqueurs territoriaux amènent à visibilité une imbrication inédite des géographies congolaises en Belgique et des temporalités belges en RDC (République démocratique du Congo, ex-Zaïre). En outre, ces narrations qui se développent en marge des circuits classiques de production de l'histoire traduisent un face à face postcolonial qui n'est pas sans continuité avec le " développement parallèle " instauré par l'ordre colonial belge au Congo.

\section{... Oral History in Matonge (Brussels): A Post-colonial Mirror}

This paper investigates the framing of memory associated with an urban district. Formerly "elite", the district of Matonge in Brussels developed at the end of the 1960s, beginning of the 1970s, when no Congolese immigration was identified in Belgium. Converting into an "African" or "multicultural" area, Matonge remains the inevitable place for Congolese meetings and festivals where "another" history can be heard. Grounding on a long-time ethnographical approach, we questioned the history of this district whose territorial frames highlight the original interweaving of Congolese geographies in Belgium and Belgian timelines in DRC (Democratic Republic of Congo, former Zaire). Besides, these narratives expand at the margins of classical circuits of history production and reveal a post-colonial face-to-face with strong continuity with the so-called "parallel development" established by the Belgian colonial order in Congo.

\section{Historia oral en Matonge (Bruselas): un espejo postcolonial}

Este artículo explora las posturas en forma memoriales asociadas con un barrio urbano. Hace poco "select», el barrio Matonge en Bruselas se desarrolló a finales de los años 1960, a principios de los años 1970, momento en el que ninguna inmigración congolesa era localizable en Bélgica. Hoy en fase de devenir "africano" o "multicultural», Matonge queda un lugar ineludible encuentros y fiestas congolesas en el curso de las cuales una "otra" historia se da a oír. A partir de un enfoque etnográfico inscrito en la duración, interrogamos la historia de este barrio (cuarto) cuyos marcadores territoriales traían a visibilidad una imbricación inédita de las geografías congolesas en Bélgica y las temporalidades belgas en RDC (la República democrática del Congo, ex-Zaire). Además, estas narraciones que se desarrollan al margen de los circuitos clásicos de producción de la historia, traducen cara a cara poscolonial que no está sin continuidad con el "desarrollo paralelo» instaurado por la orden colonial belga en el Congo. 